\title{
An Ideal OMP based Complex-Time Distribution
}

\author{
Srdjan Stanković, Irena Orović \\ Faculty of Electrical Engineering \\ University of Montenegro \\ Podgorica, Montenegro \\ e-mail:irenao@ac.me
}

\begin{abstract}
A method that provides an ideal sparse timefrequency representation, for signals with an incomplete set of samples, is proposed. It is based on the complex-time autocorrelation function and signal reconstruction algorithm performed using the orthogonal matching pursuit. The proposed method is tested on signal with fast-varying instantaneous frequency, showing remarkable performance even for a small set of input samples.
\end{abstract}

Keywords- Complex-lag distributions; compressive sensing; orthogonal matching pursuit

\section{INTRODUCTION}

The time-frequency analysis generally reveals the capability to describe the exact structure of the signal in the case of time-varying frequency content. Different timefrequency representation and distributions have been proposed for that purpose [1]-[3]. Time-frequency distributions (TFDs) provide mapping of a one-dimensional signal into a twodimensional function of time and frequency, describing the changes of the spectral content over time. Although, the timefrequency analysis was introduces more than two decades ago, the greatest developments and contributions in practical applications have been recently accomplished [3]-[7]. Namely, nowadays the time-frequency analyses are preferable in various applications, starting with the radars, sonars, communications, acoustics, data protection, etc. However, there is no an ideal time-frequency distribution that can be used for any kind of data. For instance, the spectrogram is the simplest one, but has a poor time-frequency resolution. The quadratic distributions provides high quality time-frequency representation for linear frequency modulated signals, but produce serious innerinterferences (concentration spread factor) in the case of fast varying signal phase. In order to overcome the mentioned drawbacks, polynomial and complex-time distributions of different orders have been defined [8]-[15]. Particularly, the complex-time distributions can be viewed as a Fourier transform of the complex-lag auto-correlation function. The complex-time means that the lag coordinate is scaled by the complex parameter instead of the real polynomial one as in the case of polynomial distributions. It is interesting that depending on the chosen distribution order, the concentration spread factor can be arbitrary decreased, yielding to the good concentration even for signals whose instantaneous frequency varies very fast.

The standard calculation of time-frequency distributions assumes that the whole signal is known and sampled according to the Shanon-Nyquist theorem. In accordance with the latest results in signal reconstruction theory, an important issue that arises is related to the possibility to calculate the distribution with a small reduced set of signal samples. Namely, a very popular concept used in sparse signal reconstruction is known as compressive sensing [16],[17]. If a signal is sparse in certain transform domain, then it can be compressively sampled in another (dense) domain. Then, it can be completely reconstructed using some of the signal reconstruction algorithms usually based on $l_{1}$ norm optimization methods, such as Basis Pursuit. Though the basis-pursuit algorithms represent a classical tool used to recover the incomplete signals, there are other much faster and simpler algorithms. For instance, the Orthogonal Matching Pursuit (OMP) belongs to the group of greedy algorithms that has been used for sparse approximation, and they are generally more suitable for implementation [18],[19].

In this paper we consider the OMP based approach in the calculation of the complex-lag distribution. This approach allows us to sample the signals at lower rates than those required by the sampling theorem and still to provide a highly concentrated time-frequency representation. Here, it is important to emphasize that the missing samples could occur also due to the disturbances or failures in signal processing hardware. When combined with the OMP reconstruction, the resulting complex-lag distribution is free of any interference and can be used for accurate instantaneous frequency (IF) estimation even for signal with higher order phase nonlinearities.

The paper is organized as follows. The theoretical background on the complex-time distributions is given in Section II. The OMP based complex-time distribution is proposed in Section III. The experimental evaluation is provided in Section IV, while the concluding remarks are given in Section V.

This work is supported by the Montenegrin Ministry of Science. 


\section{THEORETICAL BACKGROUND - COMPLEX-TIME DISTRIBUTIONS}

In the case of signals with highly non-stationary phase function, the concentration in the time-frequency domain depends on the rate of IF variations, as well as on the distribution order and form. Hence, the complex-lag distributions have been introduced [10]-[11] to deal with signals whose instantaneous frequency varies fast, even within a few samples. The general form of the complex-lag distribution (GCD) has been defined as:

$$
G C D_{N}(t, \omega)=\int_{-\infty}^{\infty} \prod_{k=0}^{N-1} x^{*} w_{N, k}^{*}\left(t+\frac{w_{N, k}}{N} \tau\right) e^{-j \omega \tau} d \tau
$$

where $w_{N, k}=e^{j 2 \pi k / N}$ defines the roots on the unit circle, while $N$ is the order of the distribution. It is interesting to note that for the distribution order $N=2,4,6, \ldots$, the roots on the unit circle appears in pairs:

$$
w_{N, k+N / 2}=-w_{N, k} \text {. }
$$

By increasing distribution order $N$, the influence of inner interference terms will be significantly reduced compared to the Wigner distribution. This is very important when dealing with fast varying signal's phase. However, the complexity of the correction term calculation arises with the larger $N$. The most commonly used fourth order complex-lag distribution is given in the form:

$G C D_{4}(t, \omega)=\int_{-\infty}^{\infty} x\left(t+\frac{\tau}{4}\right) x^{-1}\left(t-\frac{\tau}{4}\right) x^{-j}\left(t+j \frac{\tau}{4}\right) x^{j}\left(t-j \frac{\tau}{4}\right) e^{-j \omega \tau} d \tau$

Therefore, the distribution (2) provides significant concentration improvement with respect to the quadratic distributions, but also improvements compared to the polynomial distribution (of the same order $N=4$ ).

The discrete form of the complex-lag distribution (2) which is used in practical applications is given by:

$$
G C D_{4}(n, k)==\sum_{m=-N_{s} / 2}^{N_{s} / 2} R(n, m) e^{-j \frac{2 \pi}{N_{s}} 4 m k},
$$

where $R(n, m)$ represents the complex-lag autocorrelation function, defined as:

$$
R(n, m)=w(m) x(n+m) x^{-1}(n-m) x^{j}(n-j m) x^{-j}(n+j m),
$$

while $n, k$ and $m$ are discrete time, frequency and lag coordinate, respectively, while $N_{s}$ is the number of samples within the window $w$.

\section{OMP BASED GCD 4}

Assume that at each time point $n$ we have a small set of random samples within the auto-correlation function $R(n, m)$ defined by the random positions $q$ :

$$
\begin{gathered}
\widetilde{R}(n, m) \subset R(n, m) \\
\operatorname{card}(\widetilde{R}(n, m))<<\operatorname{card}(R(n, m))
\end{gathered} .
$$

The complex-time distribution calculated using the reduced set of samples (missing samples are replaced by zeros) would produce significant noise in the time-frequency plane. Thus, we need to recover the missing samples, and for this purpose we consider the OMP based reconstruction algorithms. Namely, if the autocorrelation function $R$ corresponds to signal form, then $R$ behaves as a locally sparse signal with one frequency component. In this case, we show that just a few samples are needed for reconstruction of complete function $R$. Moreover, by applying the OMP algorithm, an ideal timefrequency representation can be obtained for signals whose phase nonlinearity is of lower order compared to the distribution order $N$ (in our case $N=4$ is observed).

The problem of GCD calculation is recast as a problem of applying the OMP reconstruction to the measurements vector which in our case contains small number of auto-correlation samples $R$. The OMP assumes the iterative procedure where in each iteration, we select one of the columns in measurement matrix $\Phi$ that has the highest correlation with the residual part of measurements vector [18]. Then we subtract its contribution to the measurements vector and iterate on the residual that remains. For a given number of components, i.e., the number of iterations, the OMP algorithm identifies the whole set of columns that are used to obtain the sparsest approximation of $R$. The OMP based GCD calculation can be defined through the following steps:

1) Set notations: $r_{i}(n, m)$ is a residual vector ( $n$ is fixed), $\Lambda_{i}(n, m)$ is an index vector, $\widetilde{R}(n, m)$ is a measurements vector of length $M$, while $\Phi$ is a measurement matrix.

2) Set the initial residual vector as $r_{0}(n, m)=\widetilde{R}(n, m)$ and $\Lambda_{0}(n, m)=\varnothing$. The matrix $\Phi_{0}(n, m)$ is an empty matrix. The iteration counter is set to $i=1$.

3) Determine $\lambda_{i}(n, m)$ as a solution of the following minimization problem:

$$
\lambda_{i}(n, m)=\underset{j=1, \ldots, N s}{\arg \max }\left|\left\langle r_{i-1}(n, m), \phi_{j}\right\rangle\right|
$$

4) Set $\Lambda_{i}(n, m)=\Lambda_{i-1}(n, m) \cup\left\{\lambda_{i}(n, m)\right\}$

5) Set $\Phi_{i}(n, m)=\left[\Phi_{i-1}(n, m) \quad \phi_{\lambda_{i}}(n, m)\right]$ 
6) Solve the least square problem to obtain the new signal estimate:

$$
x_{i}(n, m)=\underset{x}{\arg \max }\left\|\Phi_{i}(n, m) x(n, m)-\widetilde{R}(n, m)\right\|
$$

7) Update the value of residual:

$$
r_{i}(n, m)=\widetilde{R}(n, m)-\Phi_{i}(n, m) x_{i}(n, m)
$$

8) Increment $i$, and return to Step 3 if $i<K$

9) The estimate $\hat{x}$ has non-zero indices at the components selected in $\Lambda_{K}$. The values of the estimate $\hat{x}$ in component $\lambda_{j}$ equals the $j$-th component of $x_{i}$.

10) Repeat the procedure for each time instant $n$.

The least square solution to the minimization problem given in Step 6 can be solved using the pseudo-inversion formula:

$$
x_{i}(n, m)=\left(\Phi_{i}^{T}(n, m) \Phi_{i}(n, m)\right)^{-1} \Phi_{i}^{T}(n, m) \widetilde{R}(n, m),
$$

where the matrix $\Phi_{i}$ is of size $M \times i$. The size $\Phi_{i}$ increases through the iterations, while it reaches the final size $M \mathrm{x} K$.

\section{NUMERICAL EXAMPLES}

In order to illustrate the efficiency of the proposed approach, let us consider the signal in the form:

$$
x(t)=e^{j 10 \sin (2 \pi t)+j 2 \cos (\pi t)} .
$$

The $30 \%$ of signal samples are randomly chosen and used to calculate the complex-time distribution. The standard Fourier transform based $\mathrm{GCD}_{4}$ is shown in Fig 1.a. Note that, due to $70 \%$ missing samples, the significant amount of spectral noise appear in the time-frequency domain. The same $30 \%$ of available samples are used to calculate the OMP based $\mathrm{GCD}_{4}$ shown in Fig 1.b, achieving high distribution concentration and practically an ideal time-frequency representation. Furthermore, the results shown in Fig. 2, are illustrated for a single time instant in the time-frequency domain. Note that the OMP based approach even with a quite reduced set of available samples provides the representation that corresponds to the original full data set representation.
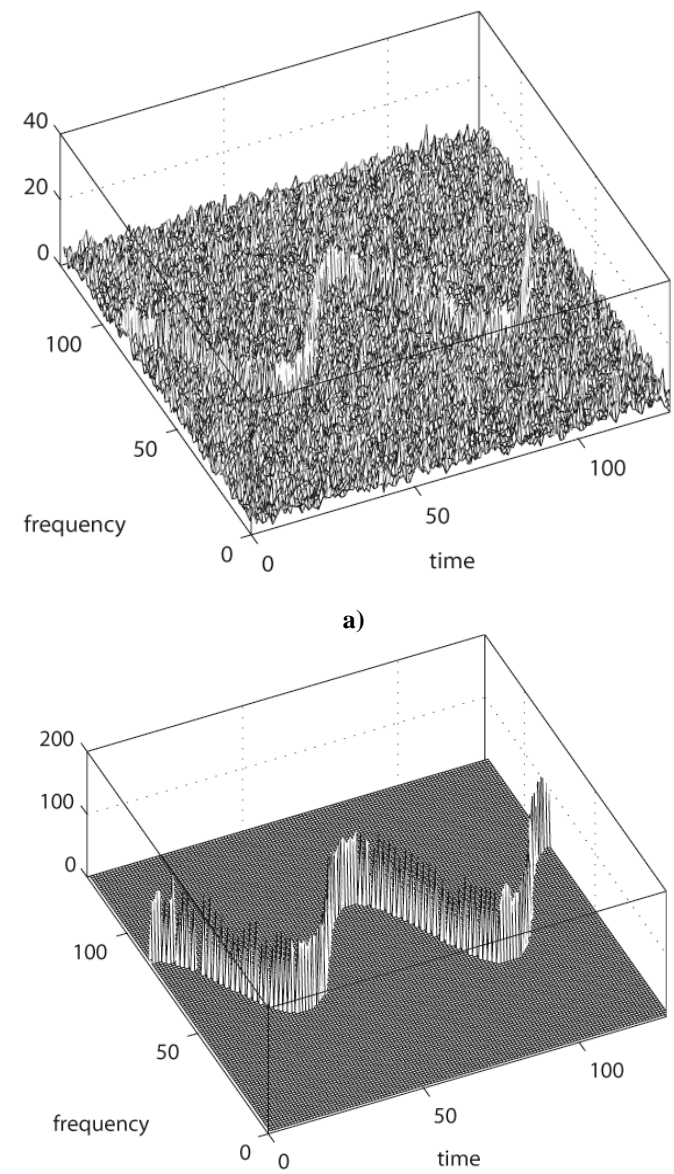

b)

Fig 1. a) Standard $\mathrm{GCD}_{4}$ calculated using $30 \%$ of samples, b) Ideal OMP based $\mathrm{GCD}_{4}$
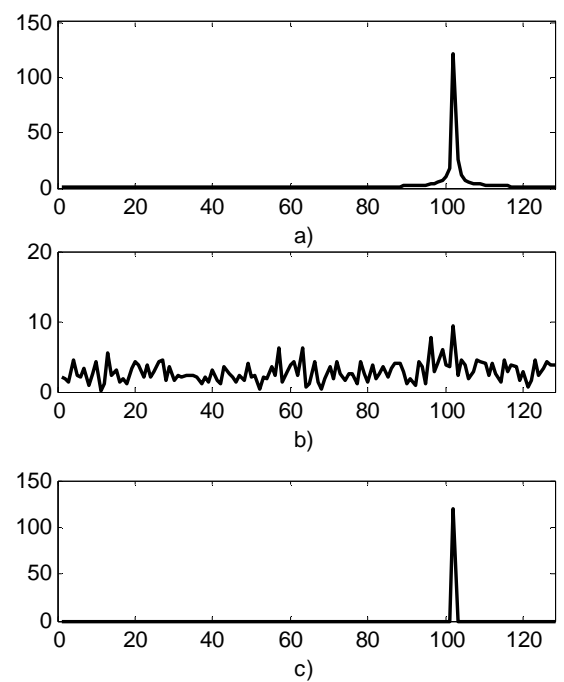

Fig 2. Single time instant: a) standard $\mathrm{CTD}_{4}$ calculated using all signal samples, b) standard $\mathrm{CTD}_{4}$ calculated using $30 \%$ of signal samples, c) OMP based ideal $\mathrm{CTD}_{4}$ calculated using $30 \%$ of signal samples 


\section{CONCLUSION}

A method for ideal time-frequency distribution calculation is proposed. The proposed method can work with a reduced set of available measurements, producing highly concentrated result as in the case of full data set. Instead of calculating the Fourier transform of incomplete auto-correlation function, the distribution is calculated using the OMP approach. Therefore, instead of noisy signal representation that results from the Fourier transform of reduced data set, we obtain an ideal representation without any disturbance.

\section{REFERENCES}

[1] B. Boashash, "Time-Frequency Analysis and Processing," Elsevier, 2003, Amsterdam

[2] L. Stanković,, M. Daković, T. Thayaparan, "Time-frequency signal analysis with applications", Artech House, Boston, 2013.

[3] J. Lerga, V. Sucic, "Nonlinear IF Estimation Based on the Pseudo WVD Adapted Using the Improved Sliding Pairwise ICI Rule," IEEE SP Letters, vol.16, no.8, pp. 953-956, 2009.

[4] E. Sejdic and J. Jiang, "'Time-Frequency Analysis of the Heart Sounds," in Proc. of 2002 ECEGRS, Ontario, Canada, May 15-17, 2002, pp. 5-9."

[5] I. Orovic, S. Stankovic, T. Thayaparan, LJ. Stankovic, "Multiwindow Smethod for Instantaneous Frequency Estimation and its Application in Radar Signal Analysis," IET Signal Processing, Vol. 4, No. 4, pp: 363370, Jan. 2010

[6] D. Petranovic, S. Stankovic, LJ. Stankovic, "Special purpose hardware for time frequency analysis," Electronics Letters, Vol.33, No.6, Mar.1997, pp.464-466.

[7] S. Stankovic, I. Djurovic, V. Vukovic, "System architecture for spacefrequency image analysis," Electronics Letters, 12.November 1998, Vol.34, No.23, pp.2224-2245
[8] X. Li, G. Bi, S. Stankovic, A. M. Zoubir, "Local polynomial Fourier transform: A review on recent developments and applications," Signal Processing, Vol. 91, No. 6, pp. 1370-1393, June, 2011

[9] B. Ristic, B. Boashash, "Relationships between the Polynomial and Higher order Wigner-Ville Distribution," IEEE Signal Processing Letters, vol. 2, no. 12, Dec. 1995, pp. 227-229.

[10] M. Morelande, B. Senadji, B. Boashash: "Complex-lag Polynomial Wigner-Ville distribution,” IEEE TENCON, 1997, pp. 43-46.

[11] S. Stanković, N. Žarić, I. Orović, C. Ioana, "General form of timefrequency distribution with complex-lag argument," Electronics Letters, vol. 44, no. 11, pp. 699-701, May 2008.

[12] S. Stanković, I. Orović and C. Ioana, "Effects of Cauchy Integral Formula Discretization on the Precision of IF Estimation: Unified Approach to Complex-Lag Distribution and its Counterpart L-Form," IEEE Signal Processing Letters, vol. 16, no. 4, April 2009., pp. 327-330.

[13] I. Orović, S. Stanković, "A Class of Highly Concentrated TimeFrequency Distributions Based on the Ambiguity Domain Representation and Complex-Lag Moment.," EURASIP Journal on Advances in Signal Processing, vol. 2009., Article ID 935314, 9 pages

[14] I. Orovic, M. Orlandic, S. Stankovic, Z. Uskokovic, "A Virtual Instrument for Time-Frequency Analysis of Signals with Highly NonStationary Instantaneous Frequency," IEEE Transactions on Instrumentation and Measurements, vol. 60, no. 3, pp. 791 - 803,2011

[15] R. Wang, Y. Jiang, "A modified fourth-order time-frequency distribution with complex-lag argument and its counterpart L-form", Consumer Electronics, Communications and Networks (CECNet), 2012 2nd International Conference on, pp: 1531 - 1535

[16] E. Candes, J. Romberg J, T. Tao T "Robust uncertainty principles: Exact signal reconstruction from highly incomplete frequency information," IEEE Trans. on Information Theory, vol. 52, no.2, 2006, pp.489-509.

[17] S. Stankovic, I. Orovic, E. Sejdic, "Compressive sensing" Ch. 6 in Multimedia Signals and Systems, Springer, 2012.

[18] J. A. Tropp, A. C. Gilbert, "Signal Recovery From Random Measurements Via Orthogonal Matching Pursuit," IEEE Transaction on Information Theory, vol. 53, no.12, 2007

[19] T. Zhang, "Sparse Recovery with Orthogonal Matching Pursuit Under RIP," IEEE Transaction on Information Theory, vol. 57, no.9, 2011 